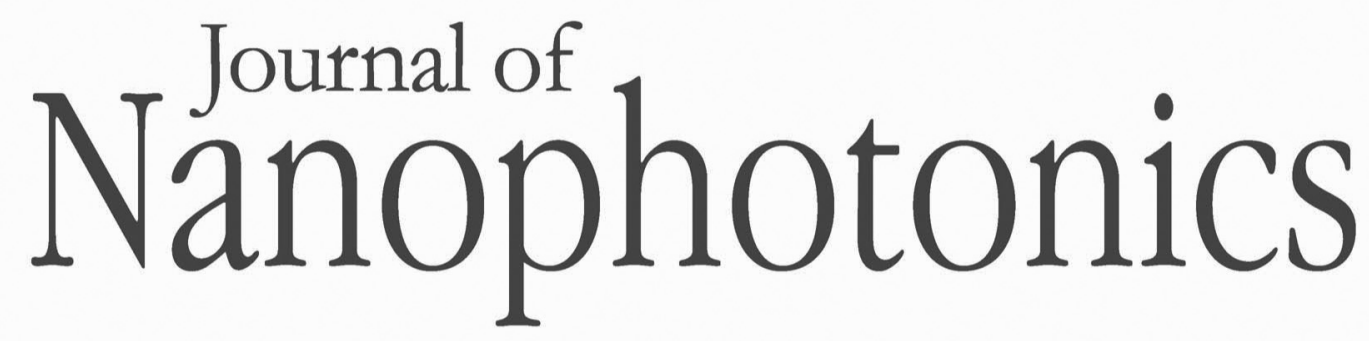

SPIEDigitalLibrary.org/jnp

\title{
Plasmonic nano-architectures for surface enhanced Raman scattering: a review
}

G. V. Pavan Kumar 


\title{
Plasmonic nano-architectures for surface enhanced Raman scattering: a review
}

\author{
G. V. Pavan Kumar \\ Indian Institute of Science Education and Research (IISER), Division of Physics and Chemistry, \\ Photonics and Optical Nanoscopy Laboratory, Pune 411008, India \\ pavan@iiserpune.ac.in
}

\begin{abstract}
Surface enhanced Raman scattering (SERS) is an optical spectroscopy technique with single molecule sensitivity and chemical specificity. The electromagnetic enhancement mechanism of SERS is facilitated by the localized surface plasmons of metallic nanostructures utilized in experiments. The magnitude of the local optical field created by the plasmonic nanostructure depends on parameters such as size, shape, morphology, arrangement, and local environment of the nanostructure. By tuning these parameters, electromagnetic hot spots can be created to facilitate ultra-sensitive, subwavelength SERS detection platforms. In recent years, there have been a number of innovations in nanofabrication and synthesis of plasmonic nanostructures. This has led to a variety of plasmonic nano-architectures that can be harnessed for SERS. Recently investigated plasmonic nanostructures in the context of SERS include nanosphere dimers, individual nanocubes, nanotriangular arrays, nano-pyramid shells, individual and assembly of nanorods, nanowires, and nanotips, and some unconventional nano-architectures. Challenges in fundamental and application aspects of SERS remain for future research. () 2012 Society of Photo-Optical Instrumentation Engineers (SPIE). [DOI: 10.1117/1.JNP.6.064503]
\end{abstract}

Keywords: surface enhanced Raman scattering; plasmonics; nano-optics; spectroscopy.

Paper 12011V received Feb. 6, 2012; revised manuscript received Mar. 16, 2012; accepted for publication Mar. 19, 2012; published online May 4, 2012.

\section{Introduction}

One of the most important aspects of nanoscale materials is that many of its physical properties can be dramatically tuned by altering its size, shape, and morphology. In recent years, various new tools and techniques have emerged to fabricate and visualize nanomaterials. ${ }^{1}$ This has led to better understanding of physical and chemical properties of nanomaterials, and has further motivated research in various directions. The preparation of nanostructures can be broadly classified in to two categories: the top-down approach and the bottom-up approach. In the top-down approach, such as electron beam lithography, ${ }^{2}$ the starting point is a bulk material which is further processed by the electron beam to create nanoscale entities. In contrast, the bottomup approach, such as chemical synthesis or self assembly, ${ }^{3}$ starts with molecular entities and builds up the nanomaterials. Each approach has its advantages and shortcomings, but can also be combined to prepare nanomaterials with unique properties. ${ }^{4,5}$

Of the many kinds of nanomaterials, metal nanostructures have been extensively studied in the context of their optical properties. ${ }^{6}$ The interaction of light with noble metals, such as silver, gold, and copper, has been a topic of research for number of years, ${ }^{6,7}$ but in last couple of decades there is a surge of interest ${ }^{8-10}$ in understanding how light interacts with metallic nanostructures. One of the main reasons for this is the plasmonic property of metallic nanostructures in and around the visible frequency spectrum. Plasmons are essentially the coupled oscillations of light and free electrons at the metal-dielectric interface. These coupled oscillations facilitate optical fields that can be used for propagation and localization of light beyond the diffraction limit. In the context of plasmons there are two fundamental excitations: surface plasmon polaritons (SPPs) and localized surface plasmons (LSPs). SPPs are propagating plasmons and can be

0091-3286/2012/\$25.00 (C) 2012 SPIE 
harnessed for various applications, including nanophotonic waveguides. ${ }^{11,12}$ LSPs facilitate localized optical fields confined to nanoscale volumes, and hence are good candidates for nano-optical sensing. ${ }^{13}$

Various optical processes of molecules such as fluorescence ${ }^{14}$ and Raman scattering ${ }^{15}$ can be influenced by the localized optical fields created by LSPs. Important aspects of molecular spectroscopy and imaging such as spectral intensity, spatial and temporal resolution can be greatly influenced with the assistance of plasmonic nanomaterials. This has led to the emergence of a variety of optical spectroscopy techniques such as metal enhanced fluorescence, ${ }^{16}$ plasmon resonance energy transfer, ${ }^{17}$ and surface enhanced Raman scattering (SERS). ${ }^{18}$ Among them, SERS has emerged as very promising spectroscopy method with single molecule sensitivity and chemical specificity.

A variety of plasmonic nanostructures can improve the capabilities of SERS towards single molecule detection limits and towards spatial resolution of few nanometres. The motivation behind this article is to review a few recently studied plasmonic nano-architectures in the context of fundamental and application aspects of SERS.

\section{Brief Overview of Surface Enhanced Raman Scattering}

Raman scattering of molecules is an inelastic scattering process whose cross-section is around $10^{-29} \mathrm{~cm}^{2} /$ molecule. ${ }^{19}$ When compared to cross-section of other optical processes, such as fluorescence $\left(10^{-19} \mathrm{~cm}^{2} /\right.$ molecule), Raman scattering is a weak phenomenon. This had hindered the usage of Raman scattering in applications where the background fluorescence was eminent. One of the effective ways to overcome this disadvantage is through surface enhanced Raman scattering (SERS). Upon adsorption of molecules on plasmonic nanostructures, the Raman signal intensity can be enhanced by many orders of magnitude. This phenomenon is generally called as surface enhanced Raman scattering ${ }^{19,20}$ and has emerged as one of the most active fields of research in plasmon enhanced optical spectroscopy.

The enhancement in the Raman signal was first observed by Fleischmann et al. ${ }^{21}$ in 1974 on roughened silver electrodes, and the increment in the Raman signal was attributed to a large number of molecules on the corrugated surface of the electrode. In 1977, two different reports by Jeanmarie et al. ${ }^{22}$ and Albercht et al. ${ }^{23}$ showed that an enhancement in the Raman signal was due to a localized electromagnetic field around the metallic nanostructure. Since then, there has been an enormous amount of work in SERS, ${ }^{15,18}$ especially in the context of its applications in molecular detection and sensing. The general consensus is that there are two important mechanisms underlying SERS. ${ }^{15,24}$ : First, and the dominant contribution towards enhancement is due to electromagnetic enhancement, ${ }^{25,26}$ where the optical field facilitated by localized plasmon resonance of metallic nanostructure increases the Raman signal intensity. The other contribution towards enhancement in SERS is due to the chemical enhancement mechanism, ${ }^{27,28}$ where the charge transfer mechanism between the adsorbed molecule and metal plays a critical role in enhancing and modifying the modes of molecular vibration.

One of the important parameters to characterize SERS substrates is to calculate the enhancement factor. ${ }^{29}$ The total enhancement in SERS is the product of incident electric field intensity enhancement $\left[\left(\mathbf{E}\left(\lambda_{\text {inc }}\right)\right]^{2}\right.$ and radiated field intensity enhancement $\left[\left(\mathbf{E}\left(\lambda_{\text {rad }}\right)\right]^{2}\right.$, where $\lambda_{\text {inc }}$ and $\lambda_{\text {rad }}$ are the incident and radiated wavelengths, respectively. If the wavelength of radiation from the SERS substrate is almost equal to the incident radiation (i.e., small values of Stokes shift) and if the losses are minimal, then the Raman enhancement factor can be approximated to be $\left[\mathbf{E}\left(\lambda_{\text {inc }}\right)\right]^{4}$. A rigorous theoretical justification for the $\left[\left(\mathbf{E}\left(\lambda_{\text {inc }}\right)\right]^{4}\right.$ enhancement can be found in Ref. 30. A good SERS substrate usually has enhancement factors ${ }^{29}$ between the values of $10^{6}$ to $10^{11}$. A comprehensive study of SERS enhancement factors can be found in Ref. 29.

Another important concept is the SERS hot spot, ${ }^{31}$ which are locations in vicinity of the plasmonic nanostructures where the local optical field is enhanced tremendously when compared to its surrounding. Any molecule in an SERS hot-spot will exhibit an enormous enhancement in its Raman scattering signal. One of the important aspects of single molecule SERS $^{32-41}$ is to localize molecules in SERS hot-spots leading to high sensitivity. 


\section{Plasmonic Nano-Architectures for SERS:}

To facilitate SERS hot-spots, plasmonic nanostructures used as SERS substrates have to be designed in such a way that their geometrical features and arrangement can lead to maximum optical field, and hence maximum Raman signal enhancement. The focus of this review is to discuss some recent developments on plasmonic nano-architectures that exhibit SERS hot-spots.

\subsection{Nanospheres}

Chemically synthesized gold and silver nanoparticles of spherical geometry are the one of the oldest and most popular substrate in SERS studies. ${ }^{25,26,28,41-45}$ Their preparation is simple, costeffective, and have been adapted for various SERS-based applications. The most common strategy to prepare them is to reduce metal salt with a relevant reducing agent and a capping agent. There are two methods of preparation of plasmonic nanoparticles that are extensively employed in SERS: 1. the Lee and Meisel method, ${ }^{42}$ which is based on citrate reduction of either $\mathrm{AgNO}_{3}$ or $\mathrm{HAuCl}_{4}$ and the resultant silver or gold nanoparticle has an average size of around $60 \mathrm{~nm}$; or 2. the Creighton method, ${ }^{43}$ which uses ice-cold sodium borohydride to reduce $\mathrm{AgNO}_{3}$ or $\mathrm{HAuCl}_{4}$ and leads to nanoparticles of average size around $20 \mathrm{~nm}$. Both these methods give excellent enhancement in Raman signature and have been employed in various SERS experiments.

Although individual nanoparticle themselves facilitate Raman enhancement, it is the junction of these nanoparticles ${ }^{46-48}$ which provide enhanced optical fields that makes single molecule SERS $^{40,41}$ based detection a possibility. This has motivated various researchers to systematically study the dimer ${ }^{32,34,37,46-52}$ and multiple junction nano-lens structures ${ }^{53}$ of plasmonic nanoparticles. The gap junction between the nanoparticles is essentially an SERS hot-spot. ${ }^{54-57}$ Any molecule in this junction is subjected to an enormous electric field, and hence their Raman scattering signals are enhanced by many orders of magnitude. In recent years, many innovative methods ${ }^{58}$ have been employed to produce plasmonic nano-dimers. For example, Li et al., ${ }^{46}$ have introduced a simple chemical synthesis method that can generate dimers of silver nanospheres [Fig. 1(a)]. Their design yielded dimers of single crystal silver nanosphere ( $30 \mathrm{~nm}$ in diameter) and with a gap of around $2 \mathrm{~nm}$. Such controlled and repeatable procedures provide well-defined systems to study SERS hot spots. The authors recorded SERS spectra of isolated nanoparticles, well-separated nanoparticles, and closely interacting dimer structures, as shown in Fig. 1(b). They observed that the molecular signature was evident only in the dimer configuration indicating that the contribution of the gap junction facilitates enhanced optical field leading to SERS signature of the molecule.

To facilitate SERS hot spots on individual nanoparticles, sharp metallic features can be incorporated on the surface of nanospheres. Recently, such nanostructure, usually called as spiked nanoparticle or nanostars, ${ }^{59-63}$ have been utilized to obtain large electric field enhancements.
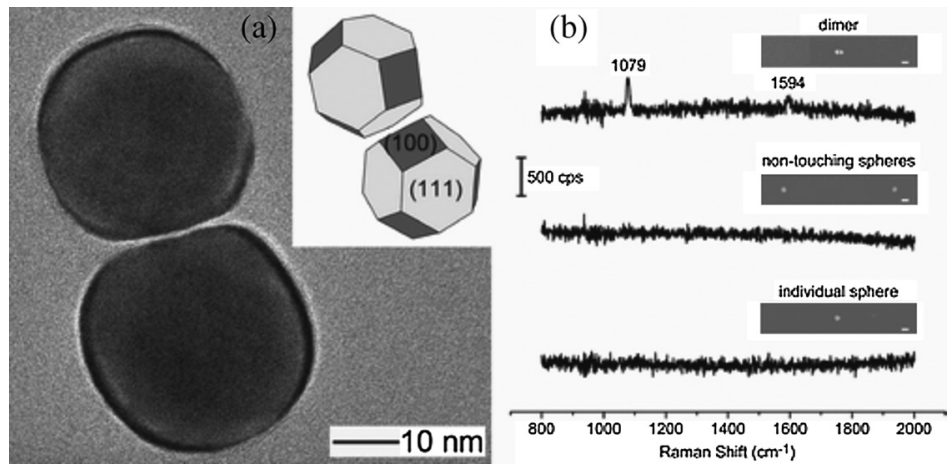

Fig. 1 (a) TEM images of silver nanosphere dimers with the inset showing a schematic illustration of nanocrystals. (b) SERS spectra of 4-methyl-benzenethiol taken from (top) a dimer of silver nanospheres, (middle) two silver nanospheres separated by $600 \mathrm{~nm}$, and (bottom) a single silver nanosphere. The scale bars in the insets correspond to $50 \mathrm{~nm}$. Reproduced with permission from Ref. 46. 
Another important category of nanoparticles are the core-shell nanostructures ${ }^{64}$ or the nanoshells. ${ }^{65-67}$ One of the major advantages of the shell-based nanoparticle is the ability to tune the plasmon resonance by tuning the physical and chemical parameters of both the shell and the core substance. ${ }^{13,68,69}$ Such nanoshell geometries have now found applications as tunable SERS substrates. ${ }^{13}$ An added advantage of this configuration is that two different kinds of nanomaterials can be incorporated as core and shell. For example, one can synthesize a nanoparticle with magnetic core and a plasmonic shell, ${ }^{70}$ thereby making the nanoparticle not only sensitive to light but also an external magnetic field. Such magnetic core metallic nanostructures can be utilized as probes for magnetic resonance imaging (MRI), which is an important diagnostic tool. Due to their versatile optical properties, nanoshells also have good potential as probes for photothermal treatment of cancer, as revealed recently. ${ }^{68}$

\subsection{Nanocubes}

Precisely shaped plasmonic nanocubes have been recently synthesized ${ }^{71-78}$ and studied in the context of SERS. One of the advantages of nanocubes is their equidistant sharp edges that facilitate highly localized optical fields, which can be harnessed to enhance Raman scattering signatures. In order to test the effect of edge sharpness, Xia and co-workers prepared isolated Ag nanocubes with sharp and truncated edges ${ }^{71}$ as shown in SEM images of Fig. 2. A thin layer of 1,4-benzenedithiol (1,4 BDT) was adsorbed on these nanocubes and a linearly polarized Raman excitation laser of $514 \mathrm{~nm}$ was incident on these individual nanocubes and the polarization was varied as shown in the Fig. 2. The Raman spectra in Fig. 2(a)-2(c) represent the polarization dependent SERS signatures from sharply-edged Ag nanocubes corresponding to the directions indicated on the SEM images. The data indicated that when the polarization is aligned along one of the edges of the nanocube, the Raman signal intensity is enhanced. In contrast, Fig. 2(d)-2(f) shows that when the edges are truncated, the SERS signature is independent of the polarization of Raman excitation. This observation was also supported by polarization dependent discrete dipole approximation calculation of near-fields, which indicated that field enhancement along the diagonal of the sharp-edged nanocubes was greater for the aligned electric field.
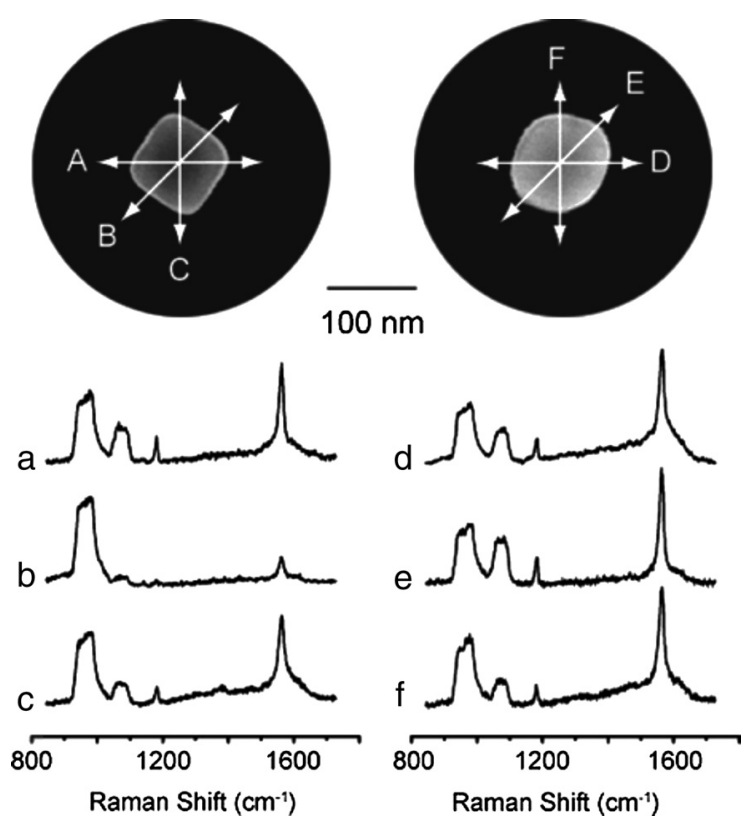

Fig. 2 Normalized SERS spectra of 1,4-BDT adsorbed on a Ag nanocube with sharp corners [left panel, (a)-(c)] and a highly truncated Ag nanocube [right panel, (d-(f)], at various angles relative to.the polarization of the excitation laser. Each SEM image shows the nanocube used and the arrows indicate the polarization directions of the incident laser corresponding to the spectra. The scale bar applies to both images. Reproduced with permission from Ref. 
Camargo et al ${ }^{74}$ have further extrapolated this work to show that SERS hot-spots can be isolated and probed by bringing two silver nanocubes close to each other. By employing a technique based on plasma etching of molecules, they isolated the molecules from all parts of the nanocube dimer except for the gap between them, thereby exclusively measuring the SERS signal between the coupled nanocubes. Such innovation can be further harnessed and applied to other plasmonic dimer nanostructures, where the SERS contribution from the gap junction is to be probed exclusively. Recently, Rycenga et al., ${ }^{78}$ have also shown that single silver nanocubes placed on metallic substrates can facilitate single molecule SERS sensitivity. Their strategy was to use coupling between the sharp edges of the nanocube with metallic layer underneath it. This coupling effectively provides a reproducible hot-spot with large field enhancement, thereby leading to ultrasensitive SERS effect on a consistent basis. Plasmon coupling effects have also been observed ${ }^{75}$ in two-dimensionally (2-D) ordered gold nanocubes on indium tin oxide substrates indicating that nanocube geometries are good substrates to study certain fundamental aspects of plasmonics and SERS.

\subsection{Nanotriangle}

Another important category of plasmonic nanostructure that has been studied in the context of SERS is nano-triangular patterns. ${ }^{79-81}$ Nanotriangles have sharp edges at their vertex which facilitate localized optical fields, and hence can be harnessed for SERS applications. One of the effective ways to produce patterns of nanotriangle pioneered by Van Dyune and co-workers ${ }^{82,83,84}$ is to employ nanosphere lithography (NSL), which is an inexpensive, versatile, and inherently parallel lithography method. Briefly, the procedure of NSL is as follows. First, size-monodisperse nanospheres are self assembled on a substrate such as glass to form a 2-D colloidal crystal deposition mask [see Fig. 3(a)]. Second, upon solvent evaporation, the desired metal is deposited either by thermal evaporation or electron beam deposition, or pulsed laser deposition through the nanosphere mask. Next, the nanosphere mask is removed by sonication of the sample in a solvent, thereby leaving behind the metal deposited in the interstitials of the nanosphere mask. A typical sample prepared using NSL is shown in Fig. 3(b). One of the major advantages of this technique is that it can be modified to vary the size, shape and arrangement of

(a)

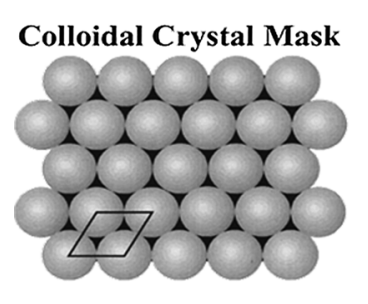

(b)

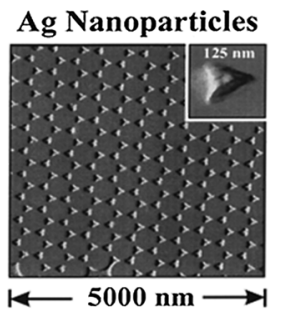

(c)

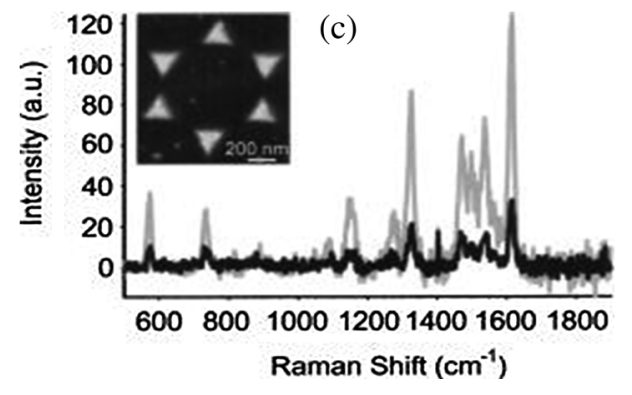

Fig. 3 Schematic illustration of a colloidal crystal mask (a) and representative AFM image (b) of Ag nanotriangular stuructures. Reproduced with permission from Ref. 82. (c) SERS enhancement achieved from R6G adsorbed on ordered $200 \mathrm{~nm}$ Ag nanocluster regions (gray trace) in comparison with amorphous Ag "film" regions (black trace) within the same sample. The signal increase is $\sim 3 \times$. Reproduced with permission from Ref. 90. 
the plasmonic nanostructures.$^{85-89}$ Schmidt et al..$^{90}$ employed NSL to study SERS properties of triangular shaped silver nanocluster arrays. They made direct comparison of rhodamine $6 \mathrm{G}$ SERS signals between ordered nanocluster region and amorphous Ag regions in the same sample [Fig. 3(c)]. The authors found that for the case of nanotriangles, the SERS signal intensity was enhanced by a factor of 3 compared to amorphous Ag regions, indicating large electromagnetic fields facilitated by the edges of the nanotriangles.

Another category similar to triangular geometry is the plasmonic nanopyramids. ${ }^{91-94}$ Stoerzinger et al. ${ }^{91}$ have recently screened individual gold nanopyramid shells and their assembly in a systematic way. The authors employed fabrication methods involving phase-shifting photolithography, etching, E-beam, and lift off (PEEL) procedures to create plasmonic nanopyramids, and by controlling the evaporation rates of the solvent of nanopyriamids, they could assemble them in three different categories: isolated single pyramids, lower order assembly, and higher order assembly (Fig. 4). They observed that as the order of assembly increased, the Raman signal intensity increased. They supported their experimental data with numerical simulations that indicated enhanced electromagnetic field between adjacent particle faces in the higher order structure. Such hierarchical nanostructures are useful to produce few-particle localized electromagnetic fields in a controlled fashion.

(a)
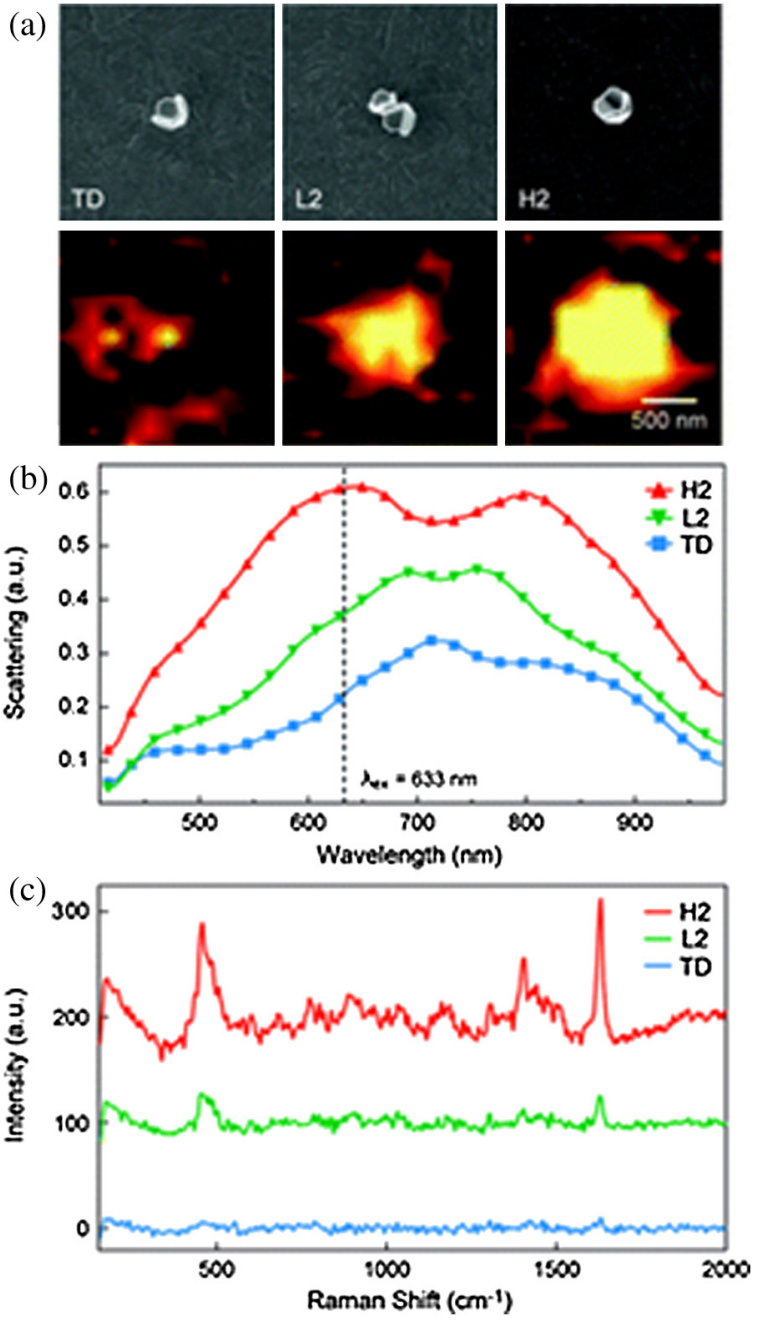

Fig. 4 (a) (top) SEM and (bottom) corresponding Raman images of the $1624 \mathrm{~cm}^{-1} \mathrm{MB}$ vibrational mode intensity from a single tip-down pyramid (TD), a low-order dimer of pyramidal nanoshells (L2), and a high-order dimer of pyramidal nanoshells (H2). The scale bar applies to all images. (b) Dark-field scattering spectra of TD, L2, and H2. (c) Raman spectra corresponding to the most intense point of the Raman image in (a). Reproduced with permission from Ref. 91. 


\subsection{Nano-Rods}

Nanorods are one of the extensively studied anisotropic plasmonic geometries. ${ }^{69,95-99}$ Various research groups have studied optical properties of gold nanorods ${ }^{69,98-102}$ and have utilized them for a variety of applications. ${ }^{97}$ Owing to their anisotropic geometry, nanorods exhibit two plasmon modes: transverse and longitudinal. ${ }^{96}$ The transverse plasmon mode is at lower wavelength and the longitudinal mode is at higher wavelength. The longitudinal plasmon mode is sensitive to the aspect ratio of the nanorod, and hence can be utilized in wavelength dependent studies. One of the important issues in plasmonics is to understand how plasmons in individual nanostructures can be coupled to obtain enhanced electromagnetic interactions. In this context, gold nanorods have been employed to study the plasmon coupling in anisotropic nanostructures. . $9,98,101,103,104^{-10}$ Kumar and Thomas ${ }^{104}$ recently investigated the SERS properties of individual nanorods and their assembly. The authors chemically couple the 111 plane at the edges of Au nanorods with linker molecules and show that the SERS signature is enhanced when the rods are in dimer geometry (Fig. 5). They further show that end-to-end coupling can be extended to form a linear chain of Au nanorods leading to an enhanced signature in Raman intensity. Such linear chains of anisotropic nanorods are good candidates to study sub-diffraction plasmonic coupling and electromagnetic transport.

Since Au nanorods are anisotropic in their geometry, they are sensitive to linear polarization of incident radiation. El-Sayed and co-workers ${ }^{105}$ have utilized this polarization dependence to show that Au nanorods conjugated with antibodies can be aligned and assembled on the surface of cancer cells. They further show that such aligned assembly of nanorods can be used as cancer diagnostic markers. Such innovations have opened up avenues for biological applications of nanorod based SERS.

\subsection{Nano-Wires}

Plasmonic nanowires are unique substrates as they support propagating plasmon polaritons. ${ }^{12,106-111}$ They sustain plasmon modes with wavelengths shorter than the incident light. Interestingly, chemically synthesized plasmonic nanowires, ${ }^{12,113}$ such as $\mathrm{Ag}$ nanowires, show minimal plasmon damping due to well-defined crystal structure, and hence have been employed in studies of plasmon polariton based waveguiding. ${ }^{111,114-118}$ In the context of SERS, coupling of an individual nanowire to another plasmonic nanostructure ${ }^{119-121}$ creates a spatially confined SERS hot spot at the junctions. One of the recent innovations in nanowire-based SERS is the remote excitation of Raman scattering. ${ }^{120,122}$ The authors have shown that the propagating plasmon property of the plasmonic nanowires can be used to perform Raman scattering at a remote location (see Fig. 6). By exciting the plasmon polariton at one end of the nanowire, a source of light can be created at the distal end of the nanowire, which further can be utilized as a Raman excitation source. The advantage of this technique is that the Raman

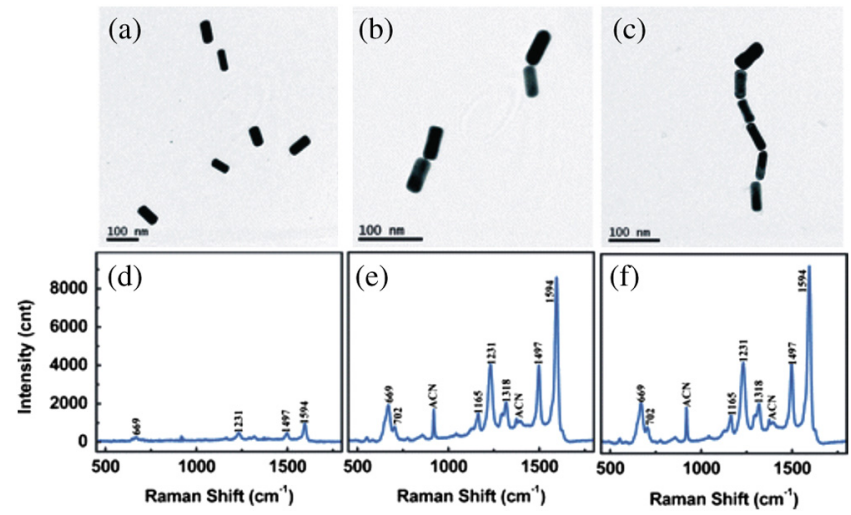

Fig. 5 TEM images of gold nanorods (a)-(c) in isolated, dimer, and chain configurations; and Raman spectrum of molecule bipy-DT (d)-(f) at various stages of plasmon coupling. Reproduced with permission from Ref. 104. 
(a)

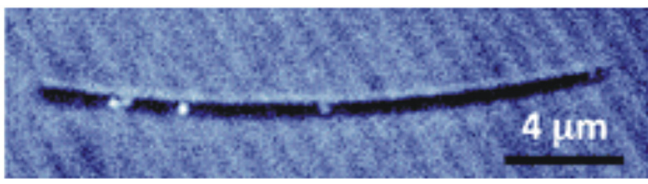

(b)

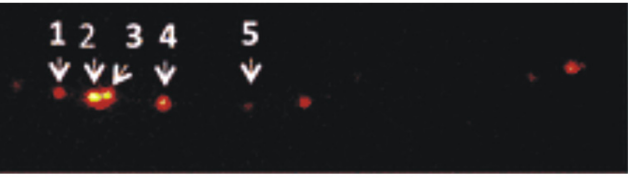

(c)

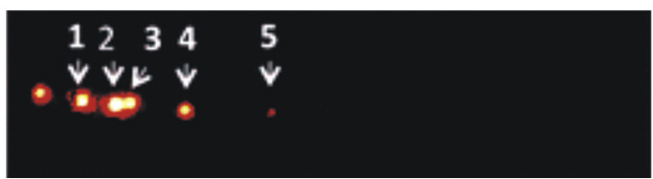

(d)

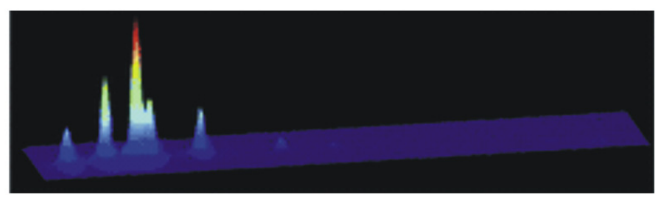

Fig. 6 Transmission (a), wide-field illumination (b), and focused laser excitation (c) images of a NPs-pATP-nanowire exhibiting seven SERS hot-spots. Focused excitation was at the left end of the wire in panel (c). (d) 3-D view of the image in panel (c). Reproduced with permission from Ref. 122.

excitation source is confined to the apex of the nanowire, and is devoid of background radiation of incident laser excitation (Fig. 6). Thus by separating the channels of excitation source and Raman scattering source, molecules in the vicinity of the distal end of the nanowire can be probed at high spatial resolution. Nanowires have also been utilized to create SERS hotspots. ${ }^{122-124}$ Recently, Chikkaraddy et al. ${ }^{124}$ have shown that by coupling two plasmonic nanowires in an end-to-end configuration, SERS hot-spots can be created at the nanowire junction. The advantage of this method is that the end-to-end coupling configuration not only facilitates SERS hot-spot, but also provides SPP based light propagation capability from one end of the nanowire pair to other distal end.

\subsection{Nanotips}

Although SERS microscopy facilitates large scale enhancement factors, it is constrained by the diffraction limit, and hence cannot spatially resolve entities smaller than the Raman excitation wavelength. Tip enhanced Raman scattering (TERS $)^{125-129}$ has recently emerged as method to perform vibrational spectroscopy beyond diffraction limit. The technique employed in TERS is essentially borrowed from apertureless near-field optical microscopy, where a sharp metallic tip is brought into close proximity of the sample deposited on the surface ${ }^{130-132}$ The tip facilitates the localized optical field which interacts with molecules beneath it, thus enhancing the Raman signal intensity. One of the important components in TERS is the morphology and composition of the tip. ${ }^{125,133-140}$ The radius of curvature of the apex of the tip determines the spatial resolution, ${ }^{134}$ and hence it is necessary to fabricate high quality plasmonic nano-tip. The most popular configuration of the tip used in TERS is a gold or silver nano-tip. ${ }^{134}$ This is usually prepared by chemical etching of wire. ${ }^{138}$ Another method to peform TERS is to employ a plasmonic nanoparticle attached to dielectric tip. ${ }^{141,142}$

In recent times, an alternative method based on adiabatic focusing of plasmons at the apex of the tip has been introduced to perform TERS by Raschke and co-workers. ${ }^{143}$ The advantage of this method is the non-local generation of nanoscale Raman excitation source which facilitates intrinsic background suppression of Raman pump beam. This was achieved by cutting a grating on a chemically etched plasmon nano-tip (Fig. 7) and exciting it with Raman pump beam. Propagating plasmons are generated due to this coupling, and further propagate towards the apex of the tip, thus creating a nanoscale light source at the discontinuity. This light source 


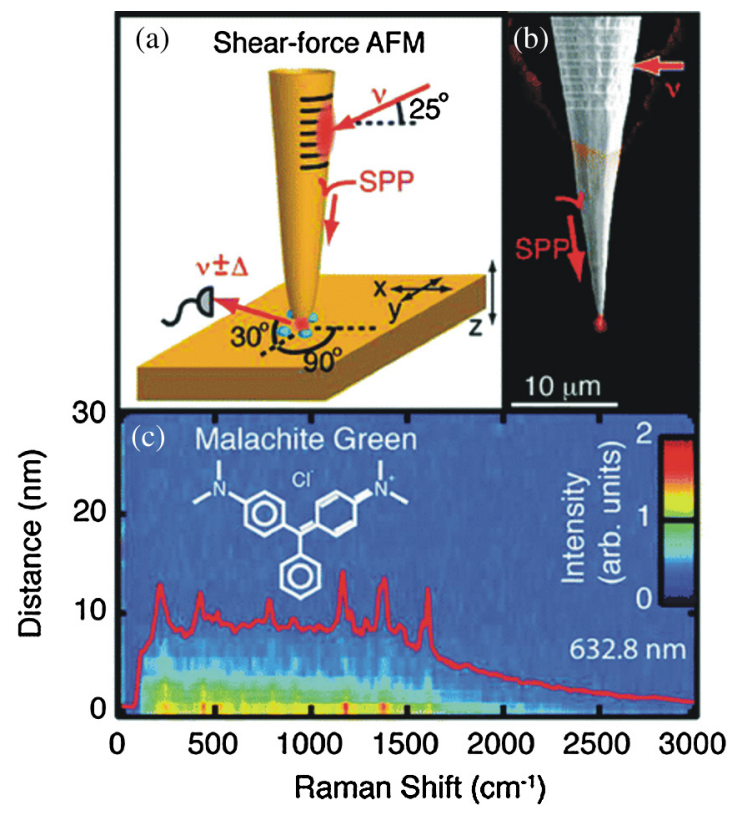

Fig. 7 (a) Schematic of the adiabatic nanofocus based the Raman scattering experiment. Electrochemically etched $\mathrm{Au}$ tips are mounted onto the quartz tuning fork of a shear-force AFM, and a grating is cut using FIB. Incident light is focused onto the grating, and the Raman scattered light excited by the nanofocused SPP at the apex is detected at a 90-deg angle. (b) SEM micrograph of an electrochemically etched Au tip superimposed with optical image of grating illumination and apex emission. (c) Grating-coupled TERS distance dependence for $\lambda=632.8 \mathrm{~nm}$ of Malachite Green on a Au surface. Reproduced with permission from Ref. 143.

can be harnessed to perform Raman spectroscopy as shown in Fig. 7(c). Adiabatic nano-focusing is an efficient method of converting far-field optical signal into near-field localized light source, ${ }^{144}$ and has immense potential to perform background-free nanoscale chemical spectroscopy and imaging. ${ }^{145-149}$

Recently, Capasso and co-workers ${ }^{150}$ have also shown that optical antenna arrays can be fabricated on an optical fiber and can be utilized as SERS micro-probes, thus expanding the prospects of in situ SERS detection.

\section{Unconventional Nano-Architectures for SERS}

Until now we have discussed plasmonic substrates that are conventionally used for SERS applications. In recent times, there have been innovations on plasmonic nanostructures designed either to probe specific issues in SERS or integrate SERS with other experimental modules. Below we briefly discuss a few cases:

- Electrostatic force based SERS-Lacharmoise et al. ${ }^{151}$ have come up with an electrochemistry based approach to guide charged molecules in a solution towards SERS active nanostructures. The authors show the possibility to selectively enhance SERS signal of various types of dye molecules according to their charge. They prepared an electrolytic cell in which charged species were selectively attracted towards SERS substrate by applying a direct current (DC) electric field. Such attracted species give rise to enhanced Raman signatures. The authors envisage their method to be useful in single molecule SERS situations where precise placement of molecules in hot-spot is a necessity.

- Shell-Isolated Nanoparticle based SERS-Although SERS is a powerful analytical technique with single molecule sensitivity, it critically depends on interaction of molecules with metallic nanostructures. Gaining control over this interaction is one of the challenges in SERS, and one of the methods towards this goal is the shell-isolated nanoparticle approach innovated by Tian and co-workers. ${ }^{152,153}$ In this method, the Raman signal is 
enhanced by gold nanoparticles with an ultrathin silica or alumina shell. In order to probe the surface of interest, a thin layer of such core-shell nanoparticle is spread as 'smart dust'. The advantages of this method are: 1. the aggregation of nanoparticles is avoided due to thin protective shell, and 2. the metallic probe is not in direct contact with the probed material. The proof of concept of this SERS technique has been shown to enhance signatures of molecules on yeast cells and citrus fruits with pesticide residues. ${ }^{152}$

- Double resonance based SERS - There are three important spectral regions in Raman spectroscopy: first is the Raman excitation region, second is the Stokes intensity region, and third is the anti-Stokes intensity region. In order to enhance the Raman intensity signatures via SERS, it would be beneficial to have plasmon resonances that overlap with these regions. By tuning the geometrical arrangement of plasmonic nanostructures, Cohen and co-workers ${ }^{154-157}$ have developed double-resonance SERS substrates. One of the methods ${ }^{154}$ to prepare double resonance substrate is to deposit gold nanodisk on $\mathrm{SiO}_{2}$ coated gold film. The gold nanodisk facilitates localized surface plasmon, and the dielectric-metal interface facilitates surface plasmon polaritons. By varying the experimental parameters, such as the geometrical arrangement of gold nanodisk and the angle of incident light, one could fabricate plasmonic substrates with double resonances. Another method ${ }^{155}$ is to use a pair of different shaped plasmonic nanostructures which inherently show plasmon resonances at different locations of the spectra. Such double resonance SERS substrates have shown to have better enhancement capabilities ${ }^{154,155}$ compared to single resonance substrates.

- SERS in optofluidic chip-Performing Raman scattering in lab-on-chip configuration has various advantages such as minimal sample requirement, compactness, and costeffectiveness. Integration of SERS with optofluidic configurations is an active area of

research. ${ }^{158-166}$ Most of the techniques either use nanoparticle-based microfluidic systems ${ }^{167-171}$ or embedded plasmonic substrates ${ }^{172,173}$ in fluidic channels to attain high sensitivity and chemical specificity. The envisaged application includes environmental monitoring, clinical diagnostics, forensic investigations, and homeland security.

\section{Future Directions and Conclusion}

SERS is now a matured research field that requires a multidisciplinary approach and fosters the combination of ideas from physics, chemistry, biology, and engineering. Although SERS is now routinely used as analytical tool for characterization of plasmonic substrates, there are some issues that require greater attention, such as:

1. Design and execution of a plasmonic substrates with consistent Raman enhancement factors.

2. Better understanding of the physics behind the SERS hot-spot mechanism.

3. Understanding the mechanism behind the chemical enhancement factor and how it can be harnessed for applications.

4. Plasmon-assisted vibrational pumping mechanism and how it can be used to get deeper insight into molecular structure and function.

Recently, there are a few developments in other research areas where SERS may play an important role. Following are a few examples:

1. There is an active search for new and better plasmonic materials. ${ }^{174}$ It has been postulated ${ }^{174,175}$ that nanomaterials made of metal oxides, noble-transition alloys, alkali-noble inter-metallic compounds, semiconductors, and graphene can exhibit plasmonic properties. Sol-gel based photonic structures ${ }^{176}$ have also been explored and shown to exhibit light management capabilities that can be adapted to perform SERS. Such materials will expand the avenues for SERS substrates and can be utilized for specific applications.

2. There has been tremendous progress made in the field of ultra-fast optics which has led to laser sources that can provide very high temporal resolutions (femto-seconds to atto-seconds). Concomitantly, there has been great progress made in scanning probe techniques that can provide spatial resolution up to a single atom. Combining both 
techniques ${ }^{177,178}$ can greatly influence the spatial and temporal resolution of chemical imaging methods, such as SERS. Non-linear Raman scattering techniques, such as coherent anti-Stokes Raman scattering (CARS) and stimulated Raman scattering can also be combined with the concepts of plasmonics. Recently, there have been a few reports ${ }^{177,179-}$ 183 that show plenty of promise in this direction.

3. Gaining new insight into the light-matter interaction has been one of the important issues in physics, and the question is how SERS can contribute to this. In recent times, SERS has been able contribute towards the understanding of ultrafast non-radiative decay rates of single molecules on metallic surfaces. ${ }^{184}$ Galloway et al. have quantified the total decay rate of single emitters close to the metallic surface, which is difficult to estimate using conventional approaches. ${ }^{184}$ In a different study, Rao et al., ${ }^{185}$ have experimentally measured mechanical forces induced by SERS on an optically trapped dielectric sphere. The authors revealed a correlation between the enhancement of the Raman signal and the average position of the trapped bead indicating momentum transfer of the emitted Raman photons on to the trapped bead.

4. Single molecule junction is one of the important components of molecular electronics that is being studied. A few research groups ${ }^{186,187}$ have employed SERS as an effective method to probe the structure of molecular junctions. Recent developments by Liu et al., ${ }^{187}$ includes a technique called as 'fishing-mode' tip enhanced Raman spectroscopy that can measure electrical conductance and Raman spectroscopy signals of a single molecule in a junction. Ward et al., ${ }^{186}$ have used SERS to determine the effective temperature of a biased metallic nano-junction decorated with molecules, which leads to the better understanding of nanoscale heating mechanisms.

Thus, we see how interaction of molecules with plasmonic nanomaterials has opened up new research avenues. Techniques such as SERS contribute to our understanding of both the plasmonic nanomaterial and the molecule interacting with it, thus making them an extremely useful tool in nano-optics and spectroscopy. In the context of nano-science and -technology, Richard Feynman had prophetically predicted that, 'There's plenty of room at the bottom'. ${ }^{188}$ Plasmonic nanomaterials combined with nano-optical techniques have shown how insightful his prediction was.

\section{Acknowledgments}

The author thanks Department of Science and Technology (DST), India for Ramanujan fellowship. This research was partially funded by DST NANOSCIENCE UNIT GRANT (SR/NM/NS42/2009) at IISER-P.

\section{References}

1. A. Biswas et al., "Advances in top-down and bottom-up surface nanofabrication: techniques, applications \& future prospects," Advances in Colloid and Interface Science in press, DOI: 10.1016/j.bbr.2011.03.031 (2012).

2. C. Vieu et al., "Electron beam lithography: resolution limits and applications," Appl. Surf. Sci. 164, 111-117 (2000), http://dx.doi.org/10.1016/S0169-4332(00)00352-4.

3. G. M. Whitesides and B. Grzybowski, "Self-assembly at all scales," Science 295, 2418-2421 (2002), http://dx.doi.org/10.1126/science.1070821.

4. B. D. Gates et al., "Unconventional nanofabrication," Annu. Rev. Mater. Res. 34, 339-372 (2004), http://dx.doi.org/10.1146/annurev.matsci.34.052803.091100.

5. P. Maury et al., in Materials Research Society Symposium Proceedings, Vol. 901, pp. 441-449, Cambridge University Press, Cambridge, UK (2005).

6. U. Kreibig and M. Vollmer, Optical Properties of Metal Clusters, Springer, Berlin (1995).

7. H. Raether, Surface Plasmons on Smooth and Rough Surfaces and on Gratings, Springer, Berlin (1988).

8. S. A. Kalele et al., "Plasmon-assisted photonics at the nanoscale," J. Nanophoton. 1, 12501 (2007), http://dx.doi.org/10.1117/1.2748429. 
9. W. L. Barnes, A. Dereux, and T. W. Ebbesen, "Surface plasmon subwavelength optics," Nature 424, 824-830 (2003), http://dx.doi.org/10.1038/nature01937.

10. V. M. Shalaev, "Electromagnetic properties of small-particle composites," Phys. Rep. 272, 61-137 (1996), http://dx.doi.org/10.1016/0370-1573(95)00076-3.

11. A. V. Zayats, II Smolyaninov, and A. A. Maradudin, "Nano-optics of surface plasmon polaritons," Phys. Rep.-Rev. Sect. Phys. Lett. 408, 131-314 (2005).

12. Surface plasmon nanophotonics; Springer Series in Optical Sciences, Vol. 131, P. G.Kik and M. L. Brongersma, ed. Springer, Dordrecht (2007).

13. S. Lal, S. Link, and N. J. Halas, "Nano-optics from sensing to waveguiding," Nat. Photon. 1, 641-648 (2007), http://dx.doi.org/10.1038/nphoton.2007.223.

14. T. H. Taminiau et al., "Optical antennas direct single-molecule emission," Nat. Photon. 2, 234-237 (2008), http://dx.doi.org/10.1038/nphoton.2008.32.

15. M. Moskovits, "Surface-enhanced spectroscopy," Rev. Mod. Phys. 57, 783-826 (1985), http://dx.doi.org/10.1103/RevModPhys.57.783.

16. C. D. Geddes and J. R. Lakowicz, "Metal-Enhanced Fluorescence," J. Fluores. 12, 121-129 (2002), http://dx.doi.org/10.1023/A:1016875709579.

17. G. L. Liu et al., "Quantized plasmon quenching dips nanospectroscopy via plasmon resonance energy transfer," Nat. Methods 4, 1015-1017 (2007), http://dx.doi.org/10.1038/ nmeth1133.

18. P. L. Stiles et al., "Surface-enhanced Raman spectroscopy," Annu. Rev. Anal. Chem. 1, 601-626 (2008), http://dx.doi.org/10.1146/annurev.anchem.1.031207.112814.

19. R. F. Aroca, Surface-Enhanced Vibrational Spectroscopy, Wiley, West Sussex (2006).

20. E. C. Le Ru and P. G. Etchegoin, Principles of Surface-Enhanced Raman Spectroscopy and Related Plasmonic Effects, Elsevier, Amsterdam (2009).

21. M. Fleischmann, P. J. Hendra, and A. J. McQuillan, "Raman spectra of pyridine adsorbed at a silver electrode," Chem. Phys. Lett. 26, 163-166 (1974), http://dx.doi.org/10.1016/ 0009-2614(74)85388-1.

22. D. L. Jeanmaire and R. P. Van Duyne, "Surface Raman spectroelectrochemistry part I. heterocyclic, aromatic, and aliphatic amines adsorbed on the anodized silver electrode," J. Electroanal. Chem. 84, 1-20 (1977), http://dx.doi.org/10.1016/S0022-0728(77)80224-6.

23. M. G. Albrecht and J. A. Creighton, "Anomalously intense Raman spectra of pyridine at a silver electrode," J. Am. Chem. Soc. 99, 5215-5217 (1977), http://dx.doi.org/10.1021/ ja00457a071.

24. G. C. Schatz, "Theoretical studies of surface enhanced Raman scattering," Accounts. Chem. Res. 17, 370-376 (1984), http://dx.doi.org/10.1021/ar00106a005.

25. M. Moskovits, "Surface-enhanced Raman spectroscopy: a brief retrospective," J. Raman Spectros. 36, 485-496 (2005), http://dx.doi.org/10.1002/(ISSN)1097-4555.

26. A. Otto et al., "Surface-enhanced Raman scattering," J. Phys.: Condens. Matt. 4, 1143-1212 (1992), http://dx.doi.org/10.1088/0953-8984/4/5/001.

27. A. Campion et al., "On the mechanism of chemical enhancement in surface-enhanced Raman scattering," J. Am. Chem. Soc. 117, 11807-11808 (1995), http://dx.doi.org/10 $.1021 / \mathrm{ja} 00152 \mathrm{a} 024$.

28. A. Campion and P. Kambhampati, "Surface-enhanced Raman scattering," Chem. Soc. Rev. 27, 241-250 (1998), http://dx.doi.org/10.1039/a827241z.

29. E. C. Le Ru et al., "Surface enhanced Raman scattering enhancement factors: a comprehensive study," J. Phys. Chem. C 111, 13794-13803 (2007), http://dx.doi.org/10.1021/ jp0687908.

30. E. C. Le Ru and P. G. Etchegoin, "Rigorous justification of the $|E| \hat{4}$ enhancement factor in surface enhanced Raman spectroscopy," Chem. Phys. Lett. 423, 63-66 (2006), http://dx .doi.org/10.1016/j.cplett.2006.03.042.

31. J. Zhao et al., "Methods for describing the electromagnetic properties of silver and gold nanoparticles," Accounts Chem. Res. 41, 1710-1720 (2008), http://dx.doi.org/10.1021/ ar800028j.

32. L. Brus, "Noble metal nanocrystals: plasmon electron transfer photochemistry and singlemolecule Raman spectroscopy," Accounts Chem. Res. 41, 1742-1749 (2008), http://dx.doi .org/10.1021/ar800121r. 
33. J.P.Camden et al., "Controlled plasmonic nanostructures for surface-enhanced spectroscopy and sensing," Accounts Chem. Res. 41, 1653-1661 (2008), http://dx.doi.org/10.1021/ ar800041s.

34. T. Dadosh et al., "Plasmonic control of the shape of the Raman spectrum of a single molecule in a silver nanoparticle dimer," ACS Nano 3, 1988-1994 (2009), http://dx.doi.org/10 $.1021 / \mathrm{nn} 900422 \mathrm{w}$.

35. J. Jiang et al., "Single molecule Raman spectroscopy at the junctions of large Ag nanocrystals," J. Phys. Chem. B 107, 9964-9972 (2003), http://dx.doi.org/10.1021/jp034632u.

36. J. R. Lombardi, R. L. Birke, and G. Haran, "Single molecule SERS spectral blinking and vibronic coupling," J. Phys. Chem. C 115, 4540-4545 (2011), http://dx.doi.org/10.1021/jp111345u.

37. J. P. Camden et al., "Probing the structure of single-molecule surface-enhanced Raman scattering hot spots," J. Am. Chem. Soc. 130, 12616-12617 (2008), http://dx.doi.org/ 10.1021/ja8051427.

38. J. A. Dieringer et al., "A frequency domain existence proof of single-molecule surfaceenhanced Raman spectroscopy," J. Am. Chem. Soc. 129, 16249-16256 (2007), http://dx .doi.org/10.1021/ja077243c.

39. E. C. Le Ru et al., "A scheme for detecting every single target molecule with surfaceenhanced raman spectroscopy," Nano Lett. 11, 5013-5019 (2011), http://dx.doi.org/10 $.1021 / \mathrm{nl} 2030344$.

40. K. Kneipp et al., "Single molecule detection using surface-enhanced Raman scattering (SERS)," Phys. Rev. Lett. 78, 1667-1670 (1997), http://dx.doi.org/10.1103/ PhysRevLett.78.1667.

41. S. Nie and S. R. Emory, "Probing single molecules and single nanoparticles by surfaceenhanced Raman scattering," Science 275, 1102-1106 (1997), http://dx.doi.org/10.1126/ science.275.5303.1102.

42. P. C. Lee and D. Meisel, "Adsorption and surface-enhanced Raman of dyes on silver and gold sols," J. Phys. Chem. 86, 3391-3395 (1982), http://dx.doi.org/10.1021/j100214a025.

43. J. A. Creighton, C. G. Blatchford, and M. G. Albrecht, "Plasma resonance enhancement of Raman scattering by pyridine adsorbed on silver or gold sol particles of size comparable to the excitation wavelength," Journal of the Chemical Society, Faraday Transactions 2: Molecular and Chemical Physics 75, 790-798 (1979).

44. M. Kerker, "The optics of colloidal silver: something old and something new," J. Colloid Interface Sci. 105, 297-314 (1985), http://dx.doi.org/10.1016/0021-9797(85)90304-2.

45. K. Kneipp et al., "Surface-enhanced Raman scattering and biophysics," J. Phys. Condens. Matter. 14, R597-R624 (2002), http://dx.doi.org/10.1088/0953-8984/14/18/202.

46. W. Li et al., "Dimers of silver nanospheres: Facile synthesis and their use as hot spots for surface-enhanced Raman scattering," Nano Lett. 9, 485-490 (2009), http://dx.doi.org/10 $.1021 / \mathrm{n} 1803621 \mathrm{x}$.

47. F. Svedberg et al., "Creating hot nanoparticle pairs for surface-enhanced Raman spectroscopy through optical manipulation," Nano Lett. 6, 2639-2641 (2006), http://dx.doi.org/10 $.1021 / \mathrm{n} 1062101 \mathrm{~m}$.

48. C. E. Talley et al., "Surface-enhanced Raman scattering from individual Au nanoparticles and nanoparticle dimer substrates," Nano Lett. 5, 1569-1574 (2005), http://dx.doi.org/10 $.1021 / \mathrm{nl} 050928 \mathrm{v}$.

49. H. Wang, C. S. Levin, and N. J. Halas, "Nanosphere arrays with controlled sub-10-nm gaps as surface-enhanced Raman spectroscopy substrates," J. Am. Chem. Soc. 127, 14992-14993 (2005), http://dx.doi.org/10.1021/ja055633y.

50. M. Rycenga et al., "Understanding the SERS effects of single silver nanoparticles and their dimers, one at a time," J. Phys. Chem. Lett. 1, 696-703 (2010), http://dx.doi.org/10.1021/ jz900286a.

51. K. L. Wustholz et al., "Structure-activity relationships in gold nanoparticle dimers and trimers for surface-enhanced Raman spectroscopy," J. Am. Chem. Soc. 132, 10903-10910 (2010), http://dx.doi.org/10.1021/ja104174m.

52. S. M. Stranahan, E. J. Titus, and K. A. Willets, "SERS orientational imaging of silver nanoparticle dimers," J. Phys. Chem. Lett. 2, 2711-2715 (2011), http://dx.doi.org/10 $.1021 /$ jz201133p. 
53. S. Bidault, F. J. GarcÃa De Abajo, and A. Polman, "Plasmon-based nanolenses assembled on a well-defined DNA template," J. Am. Chem. Soc. 130, 2750-2751 (2008), http://dx.doi .org/10.1021/ja711074n.

54. W. Rechberger et al., "Optical properties of two interacting gold nanoparticles," Opt. Commun. 220, 137-141 (2003), http://dx.doi.org/10.1016/S0030-4018(03)01357-9.

55. T. Atay, J. H. Song, and A. V. Nurmikko, "Strongly interacting plasmon nanoparticle pairs: from dipole-dipole interaction to conductively coupled regime," Nano Lett. 4, 1627-1631 (2004), http://dx.doi.org/10.1021/n1049215n.

56. J. N. Anker et al., "Biosensing with plasmonic nanosensors," Nat. Materials 7, 442-453 (2008), http://dx.doi.org/10.1038/nmat2162.

57. J. B. Lassiter et al., "Close encounters between two nanoshells," Nano Lett. 8, 1212-1218 (2008), http://dx.doi.org/10.1021/n1080271o.

58. N. J. Halas et al., "Plasmons in strongly coupled metallic nanostructures," Chem. Rev. 111, 3913-3961 (2011), http://dx.doi.org/10.1021/cr200061k.

59. C. G. Khoury and T. Vo-Dinh, "Gold nanostars for surface-enhanced Raman scattering: synthesis, characterization and optimization," J. Phys. Chem. C 112, 18849-18859 (2008).

60. P. Senthil Kumar et al., "High-yield synthesis and optical response of gold nanostars," Nanotechnology 19, 015606 (2008), http://dx.doi.org/10.1088/0957-4484/19/01/015606.

61. E. Nalbant Esenturk and A. R. Hight Walker, "Surface-enhanced Raman scattering spectroscopy via gold nanostars," J. Raman Spectros. 40, 86-91 (2009), http://dx.doi.org/10 $.1002 /$ jrs.v40:1.

62. S. Barbosa et al., "Tuning size and sensing properties in colloidal gold nanostars," Langmuir 26, 14943-14950 (2010), http://dx.doi.org/10.1021/la102559e.

63. Q. Su et al., "A reproducible SERS substrate based on electrostatically assisted APTES-functionalized surface-assembly of gold nanostars," ACS Appl. Mater. Interfaces 3, 1873-1879 (2011), http://dx.doi.org/10.1021/am200057f.

64. R. Ghosh Chaudhuri and S. Paria, "Core/shell nanoparticles: classes, properties, synthesis mechanisms, characterization, and applications," Chemical Reviews in press, DOI: $10.1021 / \mathrm{cr} 100449$ n (2012).

65. S. J. Oldenburg et al., "Nanoengineering of optical resonances," Chem. Phys. Lett. 288, 243-247 (1998), http://dx.doi.org/10.1016/S0009-2614(98)00277-2.

66. R. D. Averitt, S. L. Westcott, and N. J. Halas, "Linear optical properties of gold nanoshells," J. Opt. Soc. Am. B 16, 1824-1832 (1999), http://dx.doi.org/10.1364/JOSAB.16 .001824 .

67. J. B. Jackson and N. J. Halas, "Silver nanoshells: variations in morphologies and optical properties," J. Phys. Chem. B 105, 2743-2746 (2001), http://dx.doi.org/10.1021/ jp003868k.

68. S. Lal, S. E. Clare, and N. J. Halas, "Nanoshell-enabled photothermal cancer therapy: impending clinical impact," Accounts Chem. Res. 41, 1842-1851 (2008), http://dx.doi .org/10.1021/ar800150g.

69. S. Lal et al., "Tailoring plasmonic substrates for surface enhanced spectroscopies," Chem. Soc. Rev. 37, 898-911 (2008), http://dx.doi.org/10.1039/b705969h.

70. L. R. Hirsch et al., "Nanoshell-mediated near-infrared thermal therapy of tumors under magnetic resonance guidance," Proceedings of the National Academy of Sciences of the United States of America 100, 13549-13554 (2003), http://dx.doi.org/10.1073/pnas .2232479100 .

71. J. M. McLellan et al., "The SERS activity of a supported ag nanocube strongly depends on its orientation relative to laser polarization," Nano Lett. 7, 1013-1017 (2007), http://dx.doi .org/10.1021/n1070157q.

72. L. J. Sherry et al., "Localized surface plasmon resonance spectroscopy of single silver nanocubes," Nano Lett. 5, 2034-2038 (2005), http://dx.doi.org/10.1021/nl0515753.

73. J. M. McLellan et al., "Comparison of the surface-enhanced Raman scattering on sharp and truncated silver nanocubes," Chem. Phys. Lett. 427, 122-126 (2006), http://dx.doi.org/ 10.1016/j.cplett.2006.05.111.

74. P. H. C. Camargo et al., "Isolating and probing the hot spot formed between two silver nanocubes," Angewandte Chemie-International Edition 48, 2180-2184 (2009). 
75. H. Chen et al., "Plasmon coupling in clusters composed of two-dimensionally ordered gold nanocubes," Small 5, 2111-2119 (2009), http://dx.doi.org/10.1002/smll.v5:18.

76. J. M. McMahon et al., "Correlating the structure, optical spectra, and electrodynamics of single silver nanocubes," J. Phys. Chem. C 113, 2731-2735 (2009), http://dx.doi.org/10 $.1021 /$ jp8098736.

77. E. Ringe et al., "Unraveling the effects of size, composition, and substrate on the localized surface plasmon resonance frequencies of gold and silver nanocubes: a systematic singleparticle approach," J. Phys. Chem. C 114, 12511-12516 (2010), http://dx.doi.org/10.1021/ jp104366r.

78. M. Rycenga et al., "Generation of hot spots with silver nanocubes for single-molecule detection by surface-enhanced Raman scattering," Angewandte Chemie-International Edition 50, 5473-5477 (2011).

79. J. Zhao et al., "Localized surface plasmon resonance biosensors," Nanomedicine 1, 219-228 (2006), http://dx.doi.org/10.2217/17435889.1.2.219.

80. W. A. Murray, J. R. Suckling, and W. L. Barnes, "Overlayers on silver nanotriangles: field confinement and spectral position of localized surface plasmon resonances," Nano Lett. 6, 1772-1777 (2006), http://dx.doi.org/10.1021/n1060812e.

81. K. A. Willets and R. P. Van Duyne, "Localized surface plasmon resonance spectroscopy and sensing," Аnnu. Rev. Phys. Chem. 267-297 (2007).

82. C. L. Haynes and R. P. Van Duyne, "Nanosphere lithography: A versatile nanofabrication tool for studies of size-dependent nanoparticle optics," J. Phys. Chem. B 105, 5599-5611 (2001), http://dx.doi.org/10.1021/jp010657m.

83. J. C. Hulteen et al., "Nanosphere lithography: size-tunable silver nanoparticle and surface cluster arrays," J. Phys. Chem. B 103, 3854-3863 (1999), http://dx.doi.org/10.1021/ jp9904771.

84. X. Zhang, C. R. Yonzon, and R. P. Van Duyne, "Nanosphere lithography fabricated plasmonic materials and their applications," J. Mater. Res. 21, 1083-1092 (2006), http://dx.doi .org/10.1557/jmr.2006.0136.

85. G. L. Liu et al., "Magnetic nanocrescents as controllable surface-enhanced raman scattering nanoprobes for biomolecular imaging," Adv. Materials 17, 2683-2688 (2005), http:// dx.doi.org/10.1002/(ISSN)1521-4095.

86. J. S. Shumaker-Parry, H. Rochholz, and M. Kreiter, "Fabrication of crescent-shaped optical antennas," Adv. Materials 17, 2131-2134 (2005), http://dx.doi.org/10.1002/(ISSN) 1521-4095.

87. L. Yu et al., "Nanophotonic crescent moon structures with sharp edge for ultrasensitive biomolecular detection by local electromagnetic field enhancement effect," Nano Lett. 5, 119-124 (2005), http://dx.doi.org/10.1021/nl048232+.

88. R. Bukasov and J. S. Shumaker-Parry, "Highly tunable infrared extinction properties of gold nanocrescents," Nano Lett. 7, 1113-1118 (2007), http://dx.doi.org/10.1021/n1062317o.

89. A. Aubry et al., "Broadband plasmonic device concentrating the energy at the nanoscale: the crescent-shaped cylinder," Physical Review B-Condensed Matter and Materials Physics 82, 125430 (2010).

90. J. P. Schmidt, S. E. Cross, and S. K. Buratto, "Surface-enhanced Raman scattering from ordered Ag nanocluster arrays," J. Chem. Phys. 121, 10657-10659 (2004), http://dx.doi .org/10.1063/1.1799992.

91. K. A. Stoerzinger et al., "Screening nanopyramid assemblies to optimize surface enhanced Raman scattering," J. Phys. Chem. Lett. 1, 1046-1050 (2010), http://dx.doi.org/10.1021/ jz100095b.

92. B. Cui et al., "Fabrication of large area nanoprism arrays and their application for surface enhanced Raman spectroscopy,” Nanotechnology 19, 145302 (2008), http://dx.doi.org/10 .1088/0957-4484/19/14/145302.

93. T. H. Lin et al., "Electrochemical SERS at periodic metallic nanopyramid arrays," J. Phys. Chem. C 113, 1367-1372 (2009), http://dx.doi.org/10.1021/jp809363m.

94. C. H. Sun, N. C. Linn, and P. Jiang, "Templated fabrication of periodic metallic nanopyramid arrays," Chem. Materials 19, 4551-4556 (2007), http://dx.doi.org/10.1021/ cm0712319. 
95. B. Nikoobakht and M. A. El-Sayed, "Surface-enhanced Raman scattering studies on aggregated gold nanorods," J. Phys. Chem. A 107, 3372-3378 (2003), http://dx.doi .org/10.1021/jp026770+.

96. G. Laurent et al., "Evidence of multipolar excitations in surface enhanced Raman scattering," Physical Review B-Condensed Matter and Materials Physics 71, 1-6 (2005).

97. C. J. Murphy et al., "Anisotropic metal nanoparticles: synthesis, assembly, and optical applications," J. Phys. Chem. B 109, 13857-13870 (2005), http://dx.doi.org/10.1021/ jp0516846.

98. P. K. Jain, S. Eustis, and M. A. El-Sayed, "Plasmon coupling in nanorod assemblies: optical absorption, discrete dipole approximation simulation, and exciton-coupling model," J. Phys. Chem. B 110, 18243-18253 (2006), http://dx.doi.org/10.1021/ jp063879z.

99. R. A. Tripp, R. A. Dluhy, and Y. Zhao, "Novel nanostructures for SERS biosensing," Nano Today 3, 31-37 (2008), http://dx.doi.org/10.1016/S1748-0132(08)70042-2.

100. C. J. Orendorff et al., "Aspect ratio dependence on surface enhanced Raman scattering using silver and gold nanorod substrates," Phys. Chem. Chemical Phys. 8, 165-170 (2006), http://dx.doi.org/10.1039/b512573a.

101. S. T. Shibu Joseph et al., "Gold nanorods to nanochains: mechanistic investigations on their longitudinal assembly using $\hat{\mathrm{I}} \pm$, $\ddot{\mathrm{I}} \%$-alkanedithiols and interplasmon coupling," J. Phys. Chem. B 110, 150-157 (2006), http://dx.doi.org/10.1021/jp0544179.

102. Y. P. Zhao et al., "Polarized surface enhanced Raman and absorbance spectra of aligned silver nanorod arrays," J. Phys. Chem. B 110, 3153-3157 (2006), http://dx.doi.org/10 $.1021 /$ jp057406o.

103. P. Pramod and K. G. Thomas, "Plasmon coupling in dimers of Au nanorods," Adv. Materials 20, 4300-4305 (2008), http://dx.doi.org/10.1002/adma.v20:22.

104. J. Kumar and K. G. Thomas, "Surface-enhanced raman spectroscopy: investigations at the nanorod edges and dimer junctions," J. Phys. Chem. Lett. 2, 610-615 (2011), http://dx.doi .org/10.1021/jz2000613.

105. X. Huang et al., "Cancer cells assemble and align gold nanorods conjugated to antibodies to produce highly enhanced, sharp, and polarized surface Raman spectra: a potential cancer diagnostic marker," Nano Lett. 7, 1591-1597 (2007), http://dx.doi.org/10.1021/n1070472c.

106. R. M. Dickson and L. A. Lyon, "Unidirectional plasmon propagation in metallic nanowires," J. Phys. Chem. B 104, 6095-6098 (2000), http://dx.doi.org/10.1021/jp001435b.

107. H. Ditlbacher et al., "Silver nanowires as surface plasmon resonators," Phys. Rev. Lett. 95, 1-4 (2005), http://dx.doi.org/10.1103/PhysRevLett.95.257403.

108. A. W. Sanders et al., "Observation of plasmon propagation, redirection, and fan-out in silver nanowires," Nano Lett. 6, 1822-1826 (2006), http://dx.doi.org/10.1021/n1052471v.

109. A. V. Akimov et al., "Generation of single optical plasmons in metallic nanowires coupled to quantum dots," Nature 450, 402-406 (2007), http://dx.doi.org/10.1038/nature06230.

110. Y. Fedutik et al., "Exciton-plasmon-photon conversion in plasmonic nanostructures," Phys. Rev. Lett. 99, 136802 (2007), http://dx.doi.org/10.1103/PhysRevLett.99.136802.

111. H. Wei et al., "Cascaded logic gates in nanophotonic plasmon networks," Nat. Comm. 2, 387 (2011), http://dx.doi.org/10.1038/ncomms1388.

112. Y. Sun et al., "Crystalline silver nanowires by soft solution processing," Nano Lett. 2, 165-168 (2002), http://dx.doi.org/10.1021/n1010093y.

113. Y. Sun et al., "Uniform silver nanowires synthesis by reducing AgNO3 with ethylene glycol in the presence of seeds and poly(vinyl pyrrolidone)," Chem. Mat. 14, 4736-4745 (2002), http://dx.doi.org/10.1021/cm020587b.

114. W. Wang et al., "Light propagation in curved silver nanowire plasmonic waveguides," Nano Lett. 11, 1603-1608 (2011), http://dx.doi.org/10.1021/nl104514m.

115. C. H. Cho et al., "Tailoring hot-exciton emission and lifetimes in semiconducting nanowires via whispering-gallery nanocavity plasmons," Nat. Materials 10, 669-675 (2011), http://dx.doi.org/10.1038/nmat3067.

116. A. L. Pyayt et al., "Integration of photonic and silver nanowire plasmonic waveguides," Nat. Nanotech. 3, 660-665 (2008), http://dx.doi.org/10.1038/nnano.2008.281. 
117. Y. Ma et al., "Direct measurement of propagation losses in silver nanowires," Opt. Lett. 35, 1160-1162 (2010), http://dx.doi.org/10.1364/OL.35.001160.

118. R. Yan et al., "Direct photonic_plasmonic coupling and routing in single nanowires," Proc. Natl. Acad. Sci. USA 106, 21045-21050 (2009), http://dx.doi.org/10.1073/pnas .0902064106 .

119. J. M. Baik, S. J. Lee, and M. Moskovits, "Polarized surface-enhanced Raman spectroscopy from molecules adsorbed in nano-gaps produced by electromigration in silver nanowires," Nano Lett. 9, 672-676 (2009), http://dx.doi.org/10.1021/nl803145d.

120. Y. Fang et al., "Remote-excitation surface-enhanced Raman scattering using propagating Ag nanowire plasmons," Nano Lett. 9, 2049-2053 (2009), http://dx.doi.org/10.1021/ n1900321e.

121. J. L. Seung, M. B. Jeong, and M. Moskovits, "Polarization-dependent surface-enhanced Raman scattering from a silver-nanoparticle-decorated single silver nanowire," Nano Lett. 8, 3244-3247 (2008), http://dx.doi.org/10.1021/n1801603j.

122. J. A. Hutchison et al., "Subdiffraction limited, remote excitation of surface enhanced Raman scattering," Nano Lett. 9, 995-1001 (2009), http://dx.doi.org/10.1021/n18030696.

123. C. Du et al., "Individual Ag nanowire dimer for surface-enhanced Raman scattering," Plasmonics 6, 761-766 (2011), http://dx.doi.org/10.1007/s11468-011-9261-0.

124. R. Chikkaraddy, D. Singh, and G. V. P. Kumar, "Plasmon assisted light propagation and Raman scattering hot-spot in end-to-end coupled silver nanowire pairs," App. Phys. Lett. 100, 043108 (2012), http://dx.doi.org/10.1063/1.3679649.

125. R. Zenobi, "Analytical tools for the nano world," Analytical and Bioanalytical Chemistry 390, 215-221 (2008), http://dx.doi.org/10.1007/s00216-007-1640-1.

126. J. Stadler, T. Schmid, and R. Zenobi, "Chemical imaging on the nanoscale-top-illumination tip-enhanced Raman spectroscopy," Chimia 65, 235-239 (2011), http://dx.doi.org/10 .2533/chimia.2011.235.

127. E. Bailo and V. Deckert, "Tip-enhanced Raman scattering," Chem. Soc. Rev. 37, 921-930 (2008), http://dx.doi.org/10.1039/b705967c.

128. A. Hartschuh et al., "High-resolution near-field Raman microscopy of single-walled carbon nanotubes," Phys. Rev. Lett. 90, 095503 (2003), http://dx.doi.org/10.1103/ PhysRevLett.90.095503.

129. A. Hartschuh, N. Anderson, and L. Novotny, "Near-field Raman spectroscopy using a sharp metal tip," J. Microscopy 210, 234-240 (2003), http://dx.doi.org/10.1046/j.13652818.2003.01137.x.

130. V. Deckert et al., "Near-field surface-enhanced Raman imaging of dye-labeled DNA with 100-nm resolution," Anal. Chem. 70, 2646-2650 (1998), http://dx.doi.org/10.1021/ ac971304f.

131. D. Zeisel et al., "Near-field surface-enhanced Raman spectroscopy of dye molecules adsorbed on silver island films," Chem. Phys. Lett. 283, 381-385 (1998), http://dx.doi .org/10.1016/S0009-2614(97)01391-2.

132. R. M. Stockle et al., "Nanoscale chemical analysis by tip-enhanced Raman spectroscopy," Chem. Phys. Lett. 318, 131-136 (2000), http://dx.doi.org/10.1016/S0009-2614 (99)01451-7.

133. X. Cui et al., "Highly efficient nano-tips with metal—dielectric coatings for tip-enhanced spectroscopy applications," Chem. Phys. Lett. 453, 262-265 (2008), http://dx.doi.org/10 .1016/j.cplett.2008.01.047.

134. B. S. Yeo et al., "Tip-enhanced Raman spectroscopy-its status, challenges and future directions," Chem. Phys. Lett. 472, 1-13 (2009), http://dx.doi.org/10.1016/j.cplett.2009 .02 .023 .

135. W. Zhang et al., "Near-field heating, annealing, and signal loss in tip-enhanced Raman spectroscopy," J. Phys. Chem. C 112, 2104-2108 (2008), http://dx.doi.org/10.1021/ jp077457g.

136. E. G. Bortchagovsky and U. C. Fischer, "A tetrahedral tip as a probe for tip-enhanced Raman scattering and as a near-field Raman probe," J. Raman Spectros. 40, 1386-1391 (2009), http://dx.doi.org/10.1002/jrs.v40:10. 
137. Y. Jiang et al., "Cantilever tip near-field surface-enhanced Raman imaging of tris(bipyridine)ruthenium(ii) on silver nanoparticles-coated substrates," Langmuir 24, 12054-12061 (2008), http://dx.doi.org/10.1021/la801376p.

138. B. Ren, G. Picardi, and B. Pettinger, "Preparation of gold tips suitable for tip-enhanced Raman spectroscopy and light emission by electrochemical etching," Review of Scientific Instruments 75, 837-841 (2004), http://dx.doi.org/10.1063/1.1688442.

139. A. Tarun et al., "Highly efficient tip-enhanced Raman spectroscopy and microscopy of strained silicon," Rev. Scientific Instrum. 79, 013706 (2008), http://dx.doi.org/10.1063/ 1.2832347.

140. B. Pettinger et al., "Tip-enhanced Raman scattering: influence of the tip-surface geometry on optical resonance and enhancement," Surf. Sci. 603, 1335-1341 (2009), http://dx.doi .org/10.1016/j.susc.2008.08.033.

141. P. Bharadwaj, R. Beams, and L. Novotny, "Nanoscale spectroscopy with optical antennas," Chem. Sci. 2, 136-140 (2011), http://dx.doi.org/10.1039/c0sc00440e.

142. P. Olk et al., "Two particle enhanced nano Raman microscopy and spectroscopy," Nano Lett. 7, 1736-1740 (2007), http://dx.doi.org/10.1021/n1070727m.

143. S. Berweger et al., "Adiabatic tip-plasmon focusing for nano-Raman spectroscopy," J. Phys. Chem. Lett. 1, 3427-3432 (2010), http://dx.doi.org/10.1021/jz101289z.

144. M. I. Stockman, "Nanofocusing of optical energy in tapered plasmonic waveguides," Phys. Rev. Lett. 93, 137404 (2004), http://dx.doi.org/10.1103/PhysRevLett.93.137404.

145. F. De Angelis et al., "Breaking the diffusion limit with super-hydrophobic delivery of molecules to plasmonic nanofocusing SERS structures," Nat. Photonics 5, 682-687 (2011), http://dx.doi.org/10.1038/nphoton.2011.222.

146. C. C. Neacsu et al., "Near-field localization in plasmonic superfocusing: a nanoemitter on a tip," Nano Lett. 10, 592-596 (2010), http://dx.doi.org/10.1021/n1903574a.

147. F. De Angelis et al., "Nanoscale chemical mapping using three-dimensional adiabatic compression of surface plasmon polaritons," Nat. Nanotechnology 5, 67-72 (2010), http://dx .doi.org/10.1038/nnano.2009.348.

148. F. De Angelis et al., "A hybrid plasmonic-photonic nanodevice for label-free detection of a few molecules," Nano Lett. 8, 2321-2327 (2008), http://dx.doi.org/10.1021/nl801112e.

149. C. Ropers et al., "Grating-coupling of surface plasmons onto metallic tips: a nanoconfined light source," Nano Lett. 7, 2784-2788 (2007), http://dx.doi.org/10.1021/nl071340m.

150. E. J. Smythe et al., "Optical antenna arrays on a fiber facet for in Situ surface-enhanced Raman scattering detection," Nano Lett. 9, 1132-1138 (2009), http://dx.doi.org/10.1021/ nl803668u.

151. P. D. Lacharmoise, E. C. Le Ru, and P. G. Etchegoin, "Guiding molecules with electrostatic forces in surface enhanced raman spectroscopy," ACS Nano 3, 66-72 (2009), http:// dx.doi.org/10.1021/nn800710m.

152. J. F. Li et al., "Shell-isolated nanoparticle-enhanced Raman spectroscopy," Nature 464, 392-395 (2010), http://dx.doi.org/10.1038/nature08907.

153. J. R. Anema et al., "Shell-isolated nanoparticle-enhanced Raman spectroscopy: expanding the versatility of surface-enhanced Raman scattering," Annual Review of Analytical Chemistry 4, 129-150 (2011), http://dx.doi.org/10.1146/annurev.anchem.111808.073632.

154. Y. Chu, M. G. Banaee, and K. B. Crozier, "Double-resonance plasmon substrates for surface-enhanced raman scattering with enhancement at excitation and stokes frequencies," ACS Nano 4, 2804-2810 (2010), http://dx.doi.org/10.1021/nn901826q.

155. M. G. Banaee and K. B. Crozier, "Mixed dimer double-resonance substrates for surfaceenhanced Raman spectroscopy," ACS Nano 5, 307-314 (2011), http://dx.doi.org/10.1021/ $\mathrm{nn} 102726 \mathrm{j}$.

156. Y. Chu et al., "Double resonance surface enhanced Raman scattering substrates: an intuitive coupled oscillator model," Opt. Exp. 19, 14919-14928 (2011), http://dx.doi.org/10 .1364/OE.19.014919.

157. Y. Chu et al., "Beamed Raman: directional excitation and emission enhancement in a plasmonic crystal double resonance SERS substrate," Opt. Exp. 19, 20054-20068 (2011), http://dx.doi.org/10.1364/OE.19.020054. 
158. M. Wang et al., "An optofluidic device for surface enhanced Raman spectroscopy," Lab on a Chip-Miniaturisation for Chemistry and Biology 7, 630-632 (2007).

159. I. M. White, J. Gohring, and X. Fan, "SERS-based detection in an optofluidic ring resonator platform," Opt. Exp. 15, 17433-17442 (2007), http://dx.doi.org/10.1364/OE.15 .017433 .

160. A. Y. Lau, L. P. Lee, and J. W. Chan, "An integrated optofluidic platform for Ramanactivated cell sorting," Lab on a Chip-Miniaturisation for Chemistry and Biology 8, 1116-1120 (2008).

161. Y. S. Huh, A. J. Chung, and D. Erickson, "Surface enhanced Raman spectroscopy and its application to molecular and cellular analysis," Microfluidics and Nanofluidics 6, 285-297 (2009), http://dx.doi.org/10.1007/s10404-008-0392-3.

162. M. Wang et al., "Optofluidic device for ultra-sensitive detection of proteins using surfaceenhanced Raman spectroscopy," Microfluidics and Nanofluidics 6, 411-417 (2009), http:// dx.doi.org/10.1007/s10404-008-0397-y.

163. P. C. Ashok, B. B. Praveen, and K. Dholakia, "Near infrared spectroscopic analysis of single malt Scotch whisky on an optofluidic chip," Opt. Express 19, 22982-22992 (2011), http://dx.doi.org/10.1364/OE.19.022982.

164. C. Lim et al., "Optofluidic platforms based on surface-enhanced Raman scattering," Analyst 135, 837-844 (2010), http://dx.doi.org/10.1039/b919584j.

165. H. Gai, Y. Li, and E. S. Yeung, "Optical detection systems on microfluidic chips," Topics in Current Chemistry 304, 171-201 (2011), http://dx.doi.org/10.1007/978-3642-23050-9.

166. B. Han et al., "Application of silver-coated magnetic microspheres to a SERS-based optofluidic sensor," J. Phys. Chem. C 115, 6290-6296 (2011), http://dx.doi.org/10.1021/ jp112265e.

167. K. R. Ackermann, T. Henkel, and J. Popp, "Quantitative online detection of lowconcentrated drugs via a SERS microfluidic system," Chem. Phys. Chem. 8, 2665-2670 (2007), http://dx.doi.org/10.1002/(ISSN)1439-7641.

168. K. R. Strehle et al., "A reproducible surface-enhanced Raman spectroscopy approach. Online SERS measurements in a segmented microfluidic system," Anal. Chem. 79, 1542-1547 (2007), http://dx.doi.org/10.1021/ac0615246.

169. L. X. Quang et al., "A portable surface-enhanced Raman scattering sensor integrated with a lab-on-a-chip for field analysis," Lab on a Chip-Miniaturisation for Chemistry and Biology 8, 2214-2219 (2008).

170. Y. S. Huh et al., "Enhanced on-chip SERS based biomolecular detection using electrokinetically active microwells," Lab on a Chip_Miniaturisation for Chemistry and Biology 9, 433-439 (2009).

171. L. Tong et al., "Optical aggregation of metal nanoparticles in a microfluidic channel for surface-enhanced Raman scattering analysis," Lab on a Chip_Miniaturisation for Chemistry and Biology 9, 193-195 (2009).

172. A. Lesuffleur et al., "Apex-enhanced Raman spectroscopy using double-hole arrays in a gold film," J. Phys. Chem. C 111, 2347-2350 (2007), http://dx.doi.org/10.1021/ jp067677e.

173. R. Gordon et al., "A new generation of sensors based on extraordinary optical transmission," Accounts Chem. Res. 41, 1049-1057 (2008), http://dx.doi.org/10.1021/ar800074d.

174. P. R. West et al., "Searching for better plasmonic materials," Laser and Photonics Reviews 4, 795-808 (2010).

175. K. Biswas, S. V. Bhat, and C. N. R. Rao, "Surface-enhanced Raman spectra of azaaromatics on nanocrystals of metallic ReO3," J. Phys. Chem. C 111, 5689-5693 (2007), http://dx.doi.org/10.1021/jp068894i.

176. A. Chiappini et al., "Sol-gel-derived photonic structures: fabrication, assessment, and application,” J. Sol-Gel Sci. Tech. 60, 408-425 (2011), http://dx.doi.org/10.1007/ s10971-011-2556-y.

177. R. R. Frontiera et al., "Surface-enhanced femtosecond stimulated Raman spectroscopy," J. Phys. Chem. Lett. 2, 1199-1203 (2011), http://dx.doi.org/10.1021/jz200498z. 
178. T. Ichimura et al., "Tip-enhanced coherent anti-Stokes Raman scattering for vibrational nanoimaging," Phys. Rev. Lett. 92, 220801 (2004), http://dx.doi.org/10.1103/ PhysRevLett.92.220801.

179. C. Steuwe et al., "Surface enhanced coherent anti-Stokes Raman scattering on nanostructured gold surfaces," Nano Lett. 11, 5339-5343 (2011), http://dx.doi.org/10.1021/ nl202875w.

180. N. J. Borys and J. M. Lupton, "Surface-enhanced light emission from single hot spots in tollens reaction silver nanoparticle films: linear versus nonlinear optical excitation," J. Phys. Chem. C 115, 13645-13659 (2011), http://dx.doi.org/10.1021/jp203866g.

181. N. J. Borys, M. J. Walter, and J. M. Lupton, "Intermittency in second-harmonic radiation from plasmonic hot spots on rough silver films," Phys. Rev. B 80, 161407 (2009), http://dx .doi.org/10.1103/PhysRevB.80.161407.

182. M. J. Walter et al., "Simultaneous Raman and fluorescence spectroscopy of single conjugated polymer chains," Phys. Rev. Lett. 98, 137401 (2007), http://dx.doi.org/10.1103/ PhysRevLett.98.137401.

183. E. J. Liang et al., "Experimental observation of surface-enhanced coherent anti-Stokes Raman scattering," Chem. Phys. Lett. 227, 115-120 (1994), http://dx.doi.org/10.1016/ 0009-2614(94)00779-9.

184. C. M. Galloway, P. G. Etchegoin, and E. C. Le Ru, "Ultrafast nonradiative decay rates on metallic surfaces by comparing surface-enhanced Raman and fluorescence signals of single molecules," Phys. Rev. Lett. 103, 063003 (2009), http://dx.doi.org/10.1103/ PhysRevLett.103.063003.

185. S. Rao et al., "Measurement of mechanical forces acting on optically trapped dielectric spheres induced by surface-enhanced Raman scattering," Phys. Rev. Lett. 102, 087401 (2009), http://dx.doi.org/10.1103/PhysRevLett.102.087401.

186. D. R. Ward et al., "Vibrational and electronic heating in nanoscale junctions," Nat. Nanotech. 6, 33-38 (2011), http://dx.doi.org/10.1038/nnano.2010.240.

187. Z. Liu et al., "Revealing the molecular structure of single-molecule junctions in different conductance states by fishing-mode tip-enhanced Raman spectroscopy," Nat. Commun. 2, 305 (2011), http://dx.doi.org/10.1038/ncomms1310.

188. R. P. Feynman, "There's plenty of room at the bottom," J. Microelectromech. Syst. 1, 60-66 (1992), http://dx.doi.org/10.1109/84.128057.

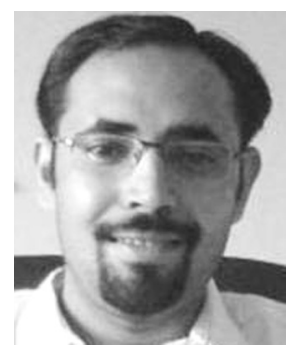

G. V. Pavan Kumar obtained his PhD from Jawaharlal Nehru Center for Advanced Scientific Research (JNCASR), Bangalore, working on Raman microscopy and its applications in plasmonics. Subsequently, he was an ICFO post-doctoral fellow at ICFO-The Institute of Photonics Sciences, Barcelona, and a post-doctoral researcher at Purdue University. In 2010, he joined the faculty of physics and chemistry at the Indian Institute of Science Education and Research (IISER), Pune, India, with Ramanujan Fellowship (DST, India). His research interests are: optical physics, multi-scale photonics, plasmonics, and Raman scattering. 